\title{
Degenerate four-wave mixing on weak transitions in the gas phase using a tunable excimer laser
}

\author{
Gerard Meijer \\ Department of Molecular and Laser Physics, University of Nijmegen, Toernooiveld, 6525 ED Nijmegen, The Netherlands
}

and

David W. Chandler

Combustion Research Facility, Sandia National Laboratories, Livermore, CA 94550, USA

Received 21 February 1992

\begin{abstract}
A simple degenerate four-wave mixing apparatus with a forward-geometry (DFWM-FG) is used to monitor weak absorptions of stable molecules in the gas phase. We demonstrate that DFWM-FG with a tunable ArF excimer laser enables the detection of $\mathrm{H}_{2}$ via the two-photon allowed $\mathrm{E}, \mathrm{F}^{1} \Sigma_{\mathrm{g}}^{+}, v^{\prime}-\mathrm{X}^{1} \Sigma_{\mathrm{g}}^{+}, v^{\prime \prime}=0$ transition and the detection of CO via the spin-forbidden a ${ }^{3} \Pi$, $v^{\prime}=2 \leftarrow \mathrm{X}^{1} \Sigma^{+}, v^{\prime \prime}=0$ transition.
\end{abstract}

Degenerate four-wave mixing (DFWM) has been demonstrated as a sensitive technique to monitor gasphase species [1-7]. The technique has been applied to $\mathrm{OH}$ and $\mathrm{NH}$ radicals where transitions to electronic states are easily accessible and to electronically excited species [8]. Related non-degenerate grating-based techniques have also recently been demonstrated by Hayden et al. [9]. These DFWM and grating based techniques complement laser-induced fluorescence, direct absorption, and ionization detection schemes. The techniques are quite sensitive and work on the absorption process; the detected molecules do not have to radiate or ionize in order to be detected. Additionally, the signal propagates as a coherent light beam and can be detected with essentially no background in a location remote from the interaction region. Similar techniques have been used to study optical phase conjugation [10,11] and to measure absorptions in liquids [12,13].

We report here the use of a simple, forward-geometry degenerate four-wave mixing (DFWM-FG) apparatus $[14,15]$ to monitor weak absorptions of stable molecules in the gas phase using a tunable ex- cimer laser. In particular we demonstrate the detection of $\mathrm{H}_{2}$ via the two-photon allowed $\mathrm{E}, \mathrm{F}^{1} \Sigma_{\mathrm{g}}^{+}, v^{\prime}$ $\leftarrow \mathrm{X}^{1} \Sigma_{\mathrm{g}}^{+} v^{\prime \prime}=0$ transition and detection of $\mathrm{CO}$ via the spin-forbidden singlet-triplet transition a ${ }^{3} \Pi$, $v^{\prime}=2 \leftarrow \mathrm{X}^{1} \Sigma^{+}, v^{\prime \prime}=0$. These spectra demonstrate the sensitivity and utility of using the tunable excimer laser in the DFWM-FG arrangement to monitor these important combustion species.

The degenerate four-wave mixing experiment with forward geometry is schematically represented in fig. 1. A mask is placed in the laser beam, to define three

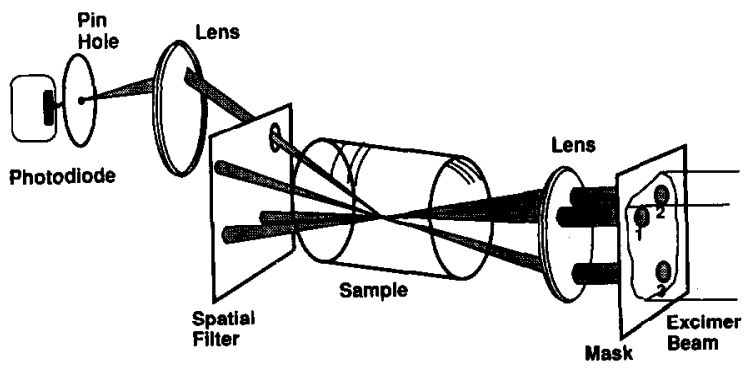

Fig. 1. Schematic view of the apparatus for a degenerate fourwave mixing with forward geometry (DFWM-FG) experiment. 
parallel coherent laser beams, at three corners of a rectangle. All three of the beams derived for this laser pulse are crossed in a cell containing the sample by focusing them through the same lens. If the wavelength of these beams is resonant with an absorption in the gas-phase sample, interference of each two of the coherent beams creates a periodically varying spatial distribution of excited molecules. The third laser beam, of the same frequency, is diffracted off this grating. The diffracted laser beam comes out of the sample at the fourth corner of the rectangle (behind the focus). The diffracted beam is degenerate with the two beams setting up the grating, both in wavelength and in time, and is automatically phasematched if the holes in the mask are chosen as described. Furthermore, it appears that in our samples the DFWM-FG observed spectral intensity pattern of the first-order diffracted light is nearly identical to the spectral intensity pattern observed in REMPI or LIF experiments on these samples, making the experimental results easily interpreted. We speculate that what is observed is the formation of density gratings from either photodissociation or from ionization of the species. At present, measurements are being performed to determine quantitatively the fluence and pressure dependencies of the signals.

It is important to note that gratings will be set up in the medium by various combinations of the incoming beams. Beam (2) and (3) will set up a grating to which beam (1) is phase-matched and thus part of beam (1) will be deflected through the spatial filter, onto the detector. Similarly, beam (1) and beam (2) will set up a grating off which part of beam (3) will deflect onto the detector, although the diffraction will not be as strong due to the larger angle of incidence. The last grating, set up by beam (1) and (3) is not detected in this geometry, however, as beam (2) does not meet the Bragg condition in this case. Beam (2) is the only beam that is present in both of the gratings that are detected, and if the intensity of this beam is kept below that of the other beams, both of the detected gratings are nearly equally intense.

In the DFWM-FG test experiments described here, a line-narrowed tunable excimer laser (Lambda Physik, EMG 150 MSCT) is used as the radiation source. The linewidth of this laser operating on ArF $(193 \mathrm{~nm})$ with unstable resonator optics in the am- plifier is about $1.0 \mathrm{~cm}^{-1}$, and the laser is tunable from $51600-51850 \mathrm{~cm}^{-1}$. The approximately $50 \mathrm{~mJ}$ energy per pulse is homogeneously distributed in a 7 by $22 \mathrm{~mm}$ rectanglar shaped beam. The laser radiation is coherent enough over the whole beam area that three parallel coherent beams can simply be formed by sending the main laser beam through a mask. The mask consists of three $2.0 \mathrm{~mm}$ diameter holes in an aluminum plate. The holes are located on three corners of a rectangle, with sides of 4.0 and $10.0 \mathrm{~mm}$. The position of the mask to the laser beam is adjusted such that the energy per pulse of the beam coming through the holes (1) and (3) is about 1.2$1.5 \mathrm{~mJ}$ whereas the beam coming through hole (2) is approximately $0.5 \mathrm{~mJ}$. All three beams are focused with a 2 inch diameter, $25 \mathrm{~cm}$ focal length lens through a quartz window in a stainless-steel cell, filled at sub-atmospheric pressures (typically between 10 and 300 mbar) with the gases that we investigated. Behind the cell a pinhole-lens-pinhole arrangement is set up to spatially filter the DFWM-FG beam, in the fourth (dark) corner of the rectangle, from the main laser beams and from scattered laser light. The signal beam is detected with a photodiode (Lambda Physik LF 302 UV); in the experiments described here the deflected laser beam is intense enough to be traced by eye via its blue fluorescence on a white piece of paper, even with the room lights on. The signal from the photodiode is sent into a boxcar integrator and the 10-shot-averaged output is displayed on a strip chart recorder.

In the upper panel of fig. 2 the DFWM-FG spectrum of the spin-forbidden one-photon transition from the $\mathrm{X}^{1} \Sigma^{+}, v^{\prime \prime}=0$ state to the metastable a ${ }^{3} \Pi$, $v^{\prime}=2$ state in CO is shown. Assignments of all the individual lines in the spectrum as well as a simulation of the expected linestrengths in direct absorption has been given elsewhere [16]. A direct comparison of the observed spectrum and the calculated spectrum is somewhat difficult. First there is the variation in the intensity of the locked, i.e. narrowband, laser power over the ArF gain profile. Around $51789.2 \mathrm{~cm}^{-1}$ for instance, narrow bandwidth laser operation ceases completely due to an intracavity metastable C-atom absorption [17]. Secondly, there are some well known rotational lines in the $4 \leftarrow 0$ band of the Schumann-Runge system of $\mathrm{O}_{2}$ that absorb to a large extent the laser light between the laser and the 


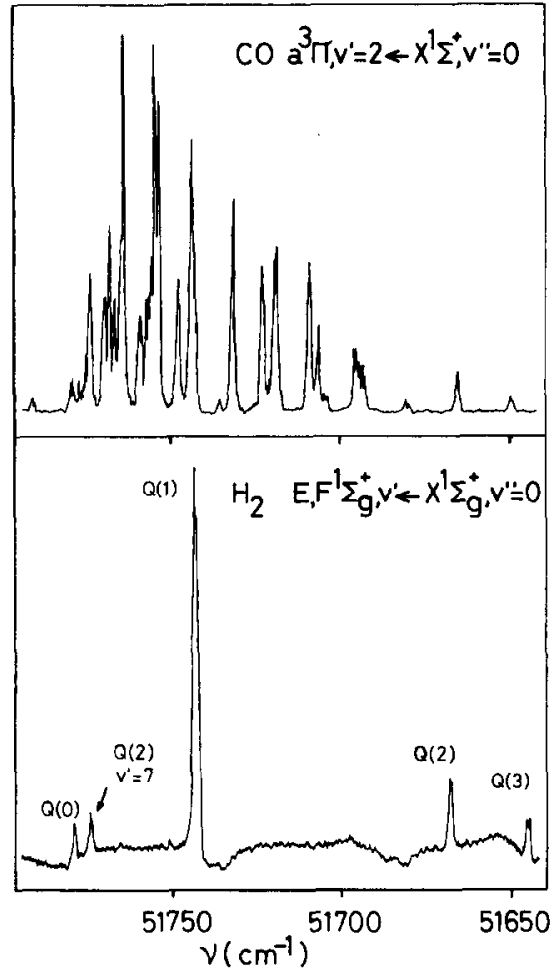

Fig. 2. DFWM-FG spectra of the spin-forbidden a ${ }^{3} \Pi, v^{\prime}=2 \leftarrow$ $\mathrm{X}^{1} \Sigma^{+}, v^{\prime \prime}=0$ transition in $300 \mathrm{mbar} \mathrm{CO}$ (upper) and of the twophoton $\mathrm{E}, \mathrm{F}^{1} \Sigma_{\mathrm{g}}^{+} v^{\prime}=6,7 \leftarrow \mathrm{X}^{1} \Sigma_{\mathrm{B}}^{+}, v^{\prime \prime}=0$ transition in $50 \mathrm{mbar} \mathrm{H}_{2}$ (lower), measured with a tunable ArF excimer laser.

detector [18]. Nevertheless, a comparison of the DFWM-FG spectrum shown here with the calculated absorption spectrum for $\mathrm{CO}$ at $300 \mathrm{~K}$ [16] shows a more or less linear correspondence between observed and calculated line intensities. The $\mathrm{CO}$ spectrum shown here was recorded with 300 mbar of $\mathrm{CO}$ in the cell, but with the photodiode $\mathrm{CO}$ spectra could be measured at pressures down to 10 mbar.

In the lower panel of fig. 2 the DFWM-FG spectrum of the $\mathrm{E}, \mathrm{F}^{1} \Sigma_{\mathrm{g}}^{+}, v^{\prime} \leftarrow \mathrm{X}^{1} \Sigma_{\mathrm{g}}^{+}, v^{\prime \prime}=0$ two-photon transition in 50 mbar of $\mathrm{H}_{2}$ is shown. The lowest Qbranch transitions to the $v^{\prime}=6$ level, as well as the $\mathrm{Q}(2)$ transition to the $v^{\prime}=7$ level fall into the tuning range of the ArF laser $[19,20]$. There is some nonresonant (but still narrow-band; if the oscillator of the tunable excimer laser is blocked the laser runs broad-band, and then this non-resonant background disappears completely) signal visible in the spectrum, possibly produced by a three-photon direct ionization ion-grating. The dips in this broad signal are due to the abovementioned $\mathrm{O}_{2}$ resonances. Essentially identical spectra to the one shown here have been obtained using either $(2+1)$-REMPI with total ion current detection [19] or two-photon excited LIF [20]. For $\mathrm{H}_{2}$ there was also a second-order diffraction signal visible, even at 50 mbar. The $\mathrm{H}_{2}$ spectrum measured via this second-order diffraction showed much different relative line intensities, however. Apart from the spectra shown in fig. 2, we also recorded DFWM-FG spectra of $\mathrm{O}_{2}$ (either in air or in 100 mbar pure $\mathrm{O}_{2}$ ), of the two-photon transition in $\mathrm{Kr}$ (at $51681.5 \mathrm{~cm}^{-1}$ ) [21] and, using the $\mathrm{KrF}$ excimer laser, of the $\widetilde{\mathrm{C}} \leftarrow \widetilde{\mathrm{X}}$ two-photon transition in $\mathrm{H}_{2} \mathrm{O}$ [22].

As is often the case in grating experiments, the exact physical origin of the signals we report here is not yet completely understood. Work is in progress to explain quantitatively the observed line intensities in the various types of beam geometries, and to determine absolute detection limits for the individual species. Application of the DFWM-FG technique to the measurement of state-dependent local densities of for instance $\mathrm{H}_{2}, \mathrm{CO}, \mathrm{O}_{2}$, and of $\mathrm{H}_{2} \mathrm{O}$ and $\mathrm{OH}$ (both with $\mathrm{KrF}$ ) in combustion processes is being investigated.

\section{Acknowledgement}

GM gratefully acknowledges the support of the Dutch Royal Academy of Science (KNAW). The visit of DC to the Nijmegen laboratory was made possible in part by financial support of the Dutch Research Institute for Materials (RIM) and the US Department of Energy, Office of Basic Energy Sciences, Division of Chemical Sciences. We thank D.H. Parker for fruitful discussions and valuable suggestions.

\section{References}

[1] P. Ewart and S.V. O'Leary, Opt. Letters 11 (1986) 279.

[2] P. Ewart and M. Kaczmarek, Appl. Opt. 30 (1991) 3996.

[3] T. Dreier and D.J. Rakestraw, Opt. Letters 15 (1990) 71.

[4] T. Dreier and D.J. Rakestraw, Appl. Phys. B 50 (1990) 479.

[5] D.J. Rakestraw, R.L. Farrow and T. Dreier, Opt. Letters 15 (1990) 709 . 
[6] T.S. Rose, W.L. Wilson, G. Wäckerle and M.D. Fayer, J. Chem. Phys. 86 (1987) 5370.

[7] J.T. Fourkas, T.R. Brewer, H. Kim and M.D. Fayer, J. Chem. Phys. 95 (1991) 5775

[8] Q. Zhang, S.A. Kandel, T.A.W. Wasserman and P.H. Vaccaro, J. Chem. Phys., in press.

[9] C. Hayden, D.W. Chandler and M.A. Buntine, to be published.

[10] R.A. Fisher, ed., Optical phase conjugation (Academic Press, New York, 1983).

[11] D.M. Pepper, Opt. Eng. 21 (1982) 155.

[12] M.D. Fayer, Ann. Rev. Phys. Chem. 33 (1982) 63.

[13] H.J. Eichler, D. Pohl and P. Gunter, Springer series in optical sciences, Vol. 50. Laser induced dynamic gratings (Springer, Berlin, 1986).
[14] G.M. Carter, J. Opt. Soc. Am. B 4 (1987) 1018.

[15] W. Du, X. Zhang, K. Chen, Z. Lu, Y. Zheng and J. Wu, Opt. Commun. 84 (1991) 205.

[16] G. Meijer, A.M. Wodtke, H. Schlüter, P. Andresen and H. Voges, J. Chem. Phys. 89 (1988) 2588.

[17] M. Versluis and G. Meijer, J. Chem. Phys., in press.

[18] D.M. Creek and R.W. Nicholls, Proc. Roy. Soc. A 341 (1975) 517.

[19] L.M. Hitchcock, G.-S. Kim, E.W. Rothe and G.P. Peck, Appl. Phys. B 52 ( 1991$) 27$.

[20] T. Okada, M. Maeda, Y. Kajiki, K. Muraoka and M. Akazaki, Appl. Phys. B 43 (1987) 113.

[21] W.K. Bischel, J. Bokor, D.J. Kliger and C.K. Rhodes, IEEE J. Quantum Electron. QE-15 (1979) 380.

[22] G. Meijer, J.J. ter Meulen, P. Andresen and A. Bath, J. Chem. Phys. 85 (1986) 6914. 